\title{
Identification and granulometric characterization of (giant) Saharan dust in sedimentary units
}

\author{
GYÖRGY VARGA ${ }^{1}$, CHRISTOPHER-BASTIAN ROETTIG ${ }^{2}$, \\ PAVLA DAGSSON-WALDHAUSEROVA ${ }^{3,4}$, FRUZSINA \\ GRESINA $^{1}$, ÁGNES ROSTÁSI ${ }^{5}$ AND JÁNOS KOVÁCS ${ }^{6}$ \\ ${ }^{1}$ Research Centre for Astronomy and Earth Sciences \\ ${ }^{2}$ Technische Universitat Dresden \\ ${ }^{3}$ Czech University of Life Sciences Prague \\ ${ }^{4}$ Agricultural University of Iceland \\ ${ }^{5}$ University of Pannonia \\ ${ }^{6}$ University of Pécs, Department of Geology \\ Presenting Author: varga.gyorgy@csfk.org
}

Mineral dust addition to sedimentary deposits and soils has a vital influence on the development and physicochemical properties of these units, and hereby the (paleo)environmental interpretation of them. A measurable amount of fine-grained Saharan dust could be incorporated into the geological sequences of areas lying further from North Africa.

Here we present our findings on Saharan dust admixture in deposits of Canary Islands, Central Europe and Iceland. The three investigation areas are influenced by Saharan dust fluxes to different degrees according to satellite observations and numerical simulations, and the differences of dust accumulation ranges cover three orders of magnitude (from some tenths to several tens of $\mathrm{g} / \mathrm{m}^{2} /$ year). The challenging identification of dust addition was achieved by developing and applying a novel method based on the direct granulometric characterization of a large number of individual mineral particles (completed with Raman spectroscopy). A Malvern Morphologi G3-ID automated static image analyzer was used to describe the size and shape properties of several tens of thousands of individual sedimentary particles per samples collected from Saharan source areas, local sedimentary units of the three investigation areas and from the material of recent Saharan dust depositional episodes.

The captured high-resolution grayscale images of individual mineral particles per samples allowed us to apply different independent approaches. Beside bulk grain size and grain shape characterization, (a) sedimentary sub-population partition by parametric curve-fitting, (b) cluster analysis, (c) Raman spectroscopy-based quartz particle identification, and (d) grayscale intensity mean values of particles were used. Interestingly, we were able to detect a relatively large number of giant $(>50 \mu \mathrm{m}$ in diameter) Saharan dust particles even in the Icelandic samples.

Support by the National Research, Development and Innovation Office (NKFIH K120620, KH130337 and PD121088) is gratefully acknowledged. The research was additionally supported by the MTA research fund KEP-08/2018. The authors would like to acknowledge the COST Action inDust (COST Action CA16202). 